\title{
PRODUÇÃO EM CONDIÇÕES SUPERCRÍTICAS E AVALIAÇÃ̃O DO POTENCIAL DO ÁCIDO BENZÓICO MICROPARTICULADO EM ALIMENTOS
}

\author{
DIEGO DIAS CARNEIRO* \\ MARIA ISABEL SOUZA OLIVEIRA** \\ MARISA FERNANDES MENDES ${ }^{\star * *}$ \\ GERSON LUIZ VIEIRA COELHO****
}

\begin{abstract}
O objetivo deste trabalho foi avaliar os melhores parâmetros da produção do ácido benzóico microparticulado (mediante aplicação do processo de expansão rápida de soluções supercríticas em diferentes condições de temperatura e pressão) e a efetividade do material obtido na inibição de microrganismos em suco de laranja natural, comparando os resultados obtidos com amostras contendo ácido benzóico comercial e com amostras sem adição de conservador por meio de análises microbiológicas. O resultado das análises indicou que a temperatura não influencia significantemente a morfologia das partículas e que na pressão de 160 bar houve a formação das menores partículas. $\mathrm{O}$ crescimento microbiológico foi mais significativo na amostra sem adição de conservador e as amostras adicionadas de 0,01 e 0,02\% m/v de ácido benzóico microparticulado apresentaram melhor controle de crescimento que as amostras com conservador comercial nas mesmas concentrações. Os resultados evidenciaram que a micronização de partículas pelo método RESS se apresenta promissora para a produção de conservadores de alimentos, porém estudos devem ser realizados para avaliar seu potencial em outros alimentos, em outros microrganismos e em outras concentrações.
\end{abstract}

PALAVRAS-CHAVE: CONSERVADORES; RESS; MICRORGANISMOS.

* Engenheiro de Alimentos, Mestrando do Programa de Pós-Graduação em Ciência e Tecnologia de Alimentos, Universidade Rural do Rio de Janeiro (UFRRJ), Seropédica, RJ (e-mail: dieguinhodc@yahoo.com.br).

** Aluna de Engenharia de Alimentos, Departamento de Tecnologia de Alimentos, UFRRJ, Seropédica, RJ (e-mail: bebelso@yahoo.com.br).

*** Professora Adjunto, Departamento de Engenharia Química, UFRRJ, Seropédica, RJ (e-mail: mendesmf@ufrrj.br).

*** Professor Titular, Departamento de Engenharia Química, UFRRJ, Seropédica, RJ (e-mail: coelho@ufrrj.br). 


\section{INTRODUÇÃO}

A necessidade de conservar os alimentos para aumentar seu tempo de prateleira e levar ao consumidor alimentos com qualidade e segurança constitui preocupação recorrente na indústria de alimentos. Isso levou o homem a introduzir diversas substancias químicas nos alimentos com ação conservadora, mas até então com propriedades desconhecidas.

Processos físicos de controle como desidratação, armazenamento a baixas temperaturas e processamento térmico são os mais utilizados na preservação de alimentos, porém sua aplicação é limitada para certos tipos de produtos. As alterações nas propriedades do alimento, o requerimento de energia do processo e a disponibilidade tecnológica tornam-se fatores limitantes no uso dos processos físicos. Com isso, em algumas situações, tais procedimentos não são adequados nem suficientes. Para esses casos, o uso de substâncias químicas com propriedades antimicrobiológicas pode ser essencial.

Os conservadores químicos atuam nos alimentos inibindo o crescimento microbiano, ou evitando reações químicas e enzimáticas, visando manter suas características iniciais por maior tempo. A relação dos conservadores com os métodos físicos de conservação torna-se complementar quando o tratamento térmico não é suficiente para destruir certos microrganismos ou suas toxinas (CALVO, 1991).

De acordo com Luck (1981), os conservadores devem apresentar baixa toxidade, permanecer estável no alimento e não alterar as suas características sensoriais. Também devem ser de fácil uso, efetivos contra os microrganismos previsíveis nas condições existentes $(\mathrm{pH}$, atividade de água, etc.) e apresentar baixo custo.

O ácido benzóico está entre os conservadores utilizados em alimentos que apresentam problemas quanto à solubilidade em meio aquoso, junto com o ácido sórbico. Sua solubilidade é de $0,29 \mathrm{~g} / 100 \mathrm{~mL}$ a $20^{\circ} \mathrm{C}$ (WHO, 2000), enquanto a do ácido sórbico é de 0,16 g/100 mL (ARAUJO, 1995).

O ácido benzóico e seus sais são utilizados mundialmente na conservação de alimentos e bebidas (WALKER, 1990). Esse ácido foi o primeiro conservador químico com uso permitido em alimentos pela Food and Drug Administration (FDA) (JAY, 2000). A quantidade de ácido benzóico a ser adicionada depende do tipo de alimento, de suas características sensoriais e da legislação do país (DE LUCA, PASSI, e QUATTRUCCI, 1995).

Atribuiu-se a atividade antimicrobiológica dos ácidos fracos, como o ácido benzóico, à sua forma não dissociada, sendo o pKa do ácido utilizado na predição de sua eficiência no alimento em determinado $\mathrm{pH}$ (ARAÚJO, 1995).

O ácido benzóico não causa danos ao ser humano quando utilizado em pequenas quantidades. O composto é imediatamente eliminado do organismo após conjugação com glicina e formação de benzoil-glicina. Essa etapa de desintoxicação impede o acumulo de ácido benzóico no corpo (FENNEMA, 1997).

Como o ácido benzóico atua nas membranas dos microrganismos, seu potencial antimicrobiano pode ser aumentado quando microparticulado, penetrando com maior facilidade em seu interior e proporcionando melhor efeito na conservação.

De acordo com Giese (1994), o ácido sórbico apresenta-se na forma de pó branco, cristalino, com baixa solubilidade em água, sendo mais solúvel em álcool (14,8 g/100 mL).

O ácido sórbico e seus sais são utilizados na conservação de produtos de laticínios, produtos de panificação, produtos do mar, sucos de frutas, vinhos, picles, maionese e molhos contra a ação de fungos, leveduras e muitas espécies de bactérias (LUECK, 1990). Seu espectro de ação antimicrobiana é relativamente amplo, particularmente contra bolores, leveduras e bactérias com reação de catalase positiva. É eficiente, portanto, na inibição da contaminação aeróbia de alimentos. No entanto, é praticamente ineficiente no controle de bactérias lácticas ou do gênero Clostridium, inclusive Clostridium botulinum (LEITÃO, 1990). 
Visando melhorar a ação dos conservadores sobre os microrganismos, aumentando sua solubilidade para que sejam gastas quantidades cada vez menores para garantir alimento seguro, deve-se submete-los ao processo que promova redução no tamanho de suas partículas.

As técnicas utilizadas para a obtenção de micropartículas são: emulsificação/evaporação do solvente, deslocamento do solvente, precipitação salina, emulsificação - difusão e fluido supercrítico (ALLEMANN, LEROUX e GURNY, 1998).

O processo de redução com fluido supercrítico, um dos mais utilizados, denomina-se Expansão Rápida de Solução Supercrítica, ou Rapid Expansion of Supercrítical (RESS). Esse processo consiste na solubilização de um soluto em fluído supercrítico e sua precipitação por meio de expansão rápida através de tubo ejetor. Importante característica desse processo consiste na formação de partículas com alto grau de pureza, estreita distribuição granulométrica e sem traços de solvente (DOMINGO, BERENDS e ROSMALEN, 1999).

Existem vários parâmetros de processo que influenciam a formação das partículas pelo processo RESS, como a temperatura e pressão na unidade de extração, a geometria do ejetor (comprimento e diâmetro), a solubilidade e a natureza da interação soluto-solvente (TURK, 1999)

O processo RESS apresenta vasta aplicação. Experimentos vêm sendo realizados em diversos tipos de materiais como, polímeros, substâncias inorgânicas e compostos farmacêuticos (FRANÇA et al., 2003). Türk et al. (2001) estudaram essas características das partículas formadas através do RESS em compostos farmacêuticos, verificando o aumento da biodisponibilidade desses agentes pouco solúveis em compostos aquosos.

Em seus estudos Helfgen et al. (2000) verificaram que o sistema em condição de alta pressão de pré-expansão e baixa temperatura de pré-expansão leva à formação de partículas de ácido benzóico com menor tamanho, aproximadamente $200 \mathrm{~nm}$ (Figura 1).

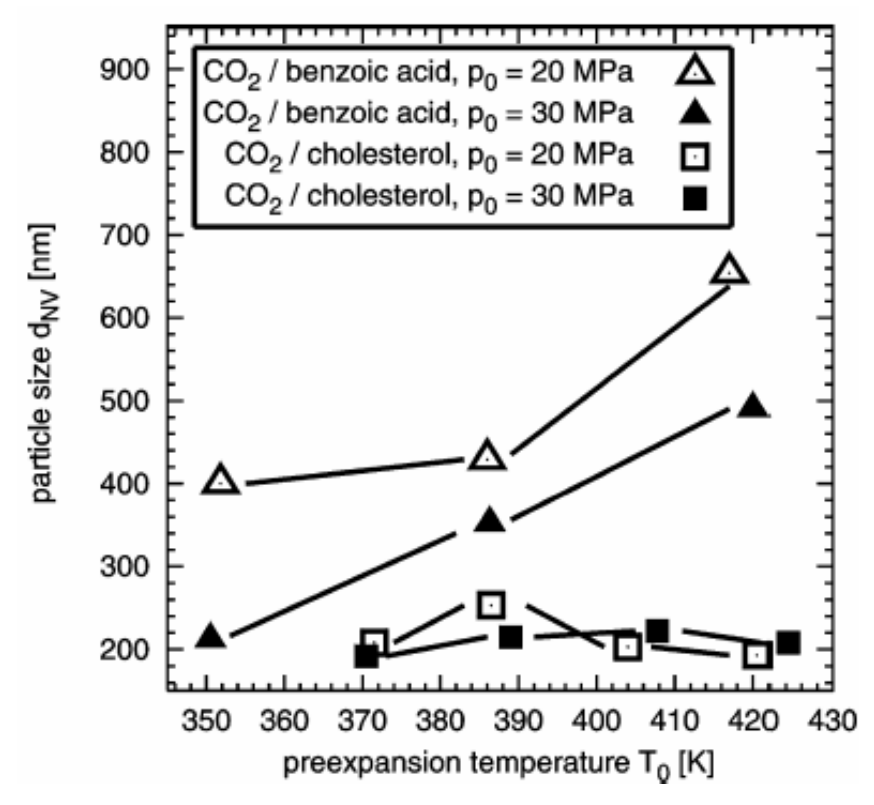

\section{FIGURA 1 - DIÂMETRO DAS PARTÍCULAS VS. CONDIÇÃO DE PRÉ-EXPANSÃO}

Fonte: Helfgen et al. 2001

O objetivo deste trabalho foi avaliar os melhores parâmetros da produção do ácido benzóico microparticulado (mediante aplicação do processo de expansão rápida de soluções supercríticas em diferentes condições de temperatura e pressão) e a efetividade do material obtido na inibição de microrganismos em suco de laranja natural, comparando os resultados obtidos com amostras contendo 
ácido benzóico comercial e com amostras sem adição de conservador por meio de análises microbiológicas.

\section{MATERIAL E MÉTODOS}

\subsection{OBTENÇÃO DAS PARTÍCULAS:}

As micropartículas de ácido benzóico foram obtidas segundo a metodologia proposta por França et al. (2004). O ácido benzóico foi solubilizado em $\mathrm{CO}_{2}$ supercrítico em vaso de pressão (reator de marca ROTH) no modo batelada, com volume de $300 \mathrm{~mL}$, provido de manômetro para o controle de pressão e banho termostático para o controle da temperatura.

Foram alimentados no reator $0,45 \mathrm{~g}$ do ácido benzóico comercial, valor esse predito pela curva de solubilidade do ácido benzóico nas condições de operação do experimento. Após a alimentação com o ácido, o reator foi fechado e em seguida conectado ao cilindro de gás para a primeira injeção de $\mathrm{CO}_{2}$ Com a pressão no reator estabilizada, o cilindro foi fechado e após a liberação de todo o gás no conector o reator foi removido e levado para resfriamento por aproximadamente uma hora no congelador. Passado o período de resfriamento, o reator foi levado novamente ao cilindro de gás para a segunda injeção de $\mathrm{CO}_{2}$. Essa fase de resfriamento contribui para melhorar a efetividade do processo, pois a redução no volume do gás contido do interior no reator permite a adição de maior quantidade de $\mathrm{CO}_{2}$ no reator, possibilitando que as condições supercríticas sejam atingidas com menores temperaturas.

Os experimentos foram conduzidos nas pressões de 120 e 160 bar com temperatura variando entre $35-50^{\circ} \mathrm{C}$. Quando o manômetro estabilizou na pressão desejada foi necessário aguardar, aproximadamente, 15 minutos para ocorrer equilíbrio entre o solvente e o soluto naquelas condições.

Para a obtenção das micropartículas, a válvula de expansão foi aberta por curto período de tempo diretamente para a câmara de coleta. A válvula de expansão deve estar envolvida por uma manta de aquecimento com temperatura pouco superior à temperatura do interior do reator para evitar a formação de cristal no interior da válvula de expansão. O esquema do sistema de alta pressão pode ser observado na Figura 2.

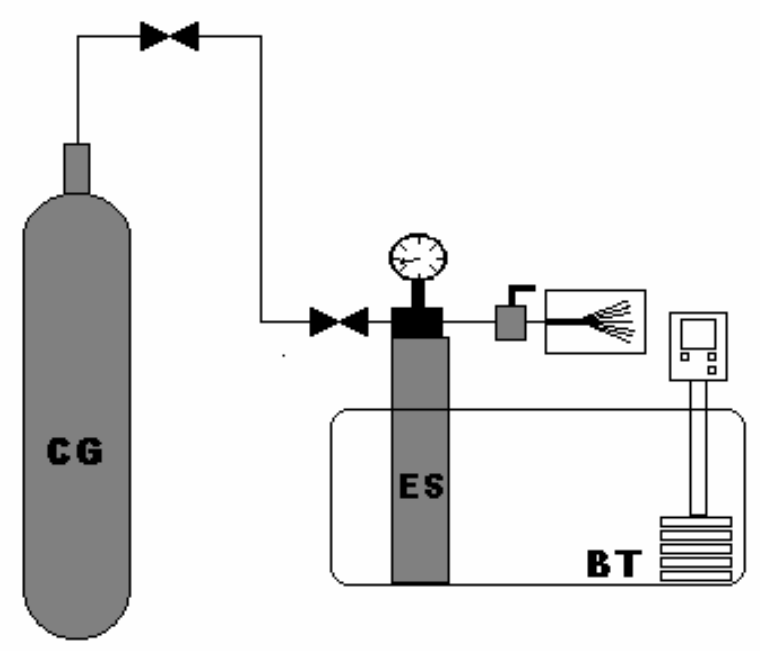

FIGURA 2 - UNIDADE DE EXTRAÇÃO SUPERCRÍTICA 


\subsection{MORFOLOGIADAS PARTÍCULAS}

O material coletado foi submetido à análise em microscópio eletrônico de varredura para verificar em qual condição de pré-expansão foram obtidas as menores partículas (França et al., 2004).

\subsection{AVALIAÇÃO DO POTENCIAL CONSERVADOR}

O potencial do conservador microparticulado foi avaliado pelo acompanhamento do crescimento de bolores e leveduras em suco de laranja natural não pasteurizado, distribuído em cinco lotes distintos: sem conservador, adicionado de conservador comercial $0,01 \% \mathrm{~m} / \mathrm{v}$, adicionado de conservador comercial $0,02 \% \mathrm{~m} / \mathrm{v}$, adicionado de conservador microparticulado $0,01 \% \mathrm{~m} / \mathrm{v}$ e adicionado de conservador comercial $0,02 \% \mathrm{~m} / \mathrm{v}$. Usou-se suco de laranja pois, além de apresentar valor de $\mathrm{pH}$ baixo (que favorece o potencial desse conservador), é ótimo substrato para o crescimento de bolores e leveduras (microrganismos mais afetados por esse conservador). Essa análise foi realizada de acordo com a metodologia proposta por pela APHA (1992), considerando que a Agência Nacional de Vigilância Sanitária (RDC 12) não estabelece limites para bolores e leveduras em suco de laranja (BRASIL, 2001). Estudouse a vida-de-prateleira do suco por 21 dias, sendo retiradas alíquotas semanalmente, em triplicata, de cada um dos lotes para serem incubadas em placas contendo ágar batata dextrose durante 5 dias em temperatura ambiente. Após esse período realizou-se a contagem microbiana para comparar o crescimento de bolores e leveduras entre os lotes.

\section{RESULTADOS E DISCUSSÃO}

Como pode ser observado na Tabela 1, as menores partículas foram obtidas nas condições de pré-expansão com maiores pressões e menores temperaturas, resultado que condiz com o informado pela literatura (HELFGEN et al., 2000).

TABELA 1 - CONDIÇÕES DE OPERAÇÃO E MORFOLOGIA DOS CRISTAIS

Condições de Pré-Expansão

$\mathrm{T}\left({ }^{\circ} \mathrm{C}\right)$

43,8

46,9

38,8

49,2

42,7
$\mathrm{P}$ (bar)

$$
\begin{aligned}
& 120 \\
& 120 \\
& 160 \\
& 160 \\
& 160
\end{aligned}
$$

Características dos Cristais

$$
\begin{aligned}
& \text { Partículas porosas }(30-50 \mu \mathrm{m}) \\
& \text { Aglomerados }(50-70 \mu \mathrm{m}) \\
& \text { Cristais em bastões }(5-10 \mu \mathrm{m}) \\
& \text { Partículas porosas }(30-60 \mu \mathrm{m}) \\
& \text { Cristais em bastões }(5-10 \mu \mathrm{m})
\end{aligned}
$$

A diferença na morfologia do ácido benzóico comercial e microparticulado pode ser observada nas Figuras 3 e 4.

As Figuras 3 e 4 mostram de forma clara que após o processo RESS, o material apresenta melhor distribuição granulométrica.

Os materiais microparticulados utilizados na avaliação do potencial do conservador foram os obtidos em 160 bar, condição de pressão que promoveu a menor granulometria. 
As boas condições higiênico-sanitárias no preparo das amostras de suco de laranja foram comprovadas pelos resultados negativos nos testes de coliformes totais e salmonela spp. realizados.

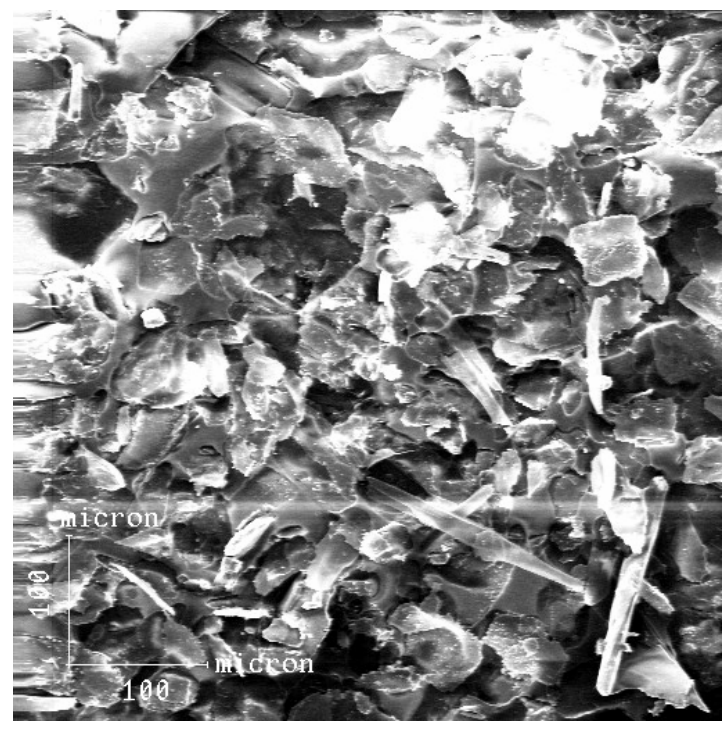

FIGURA 3 - MORFOLOGIA DO ÁCIDO BENZÓICO COMERCIAL

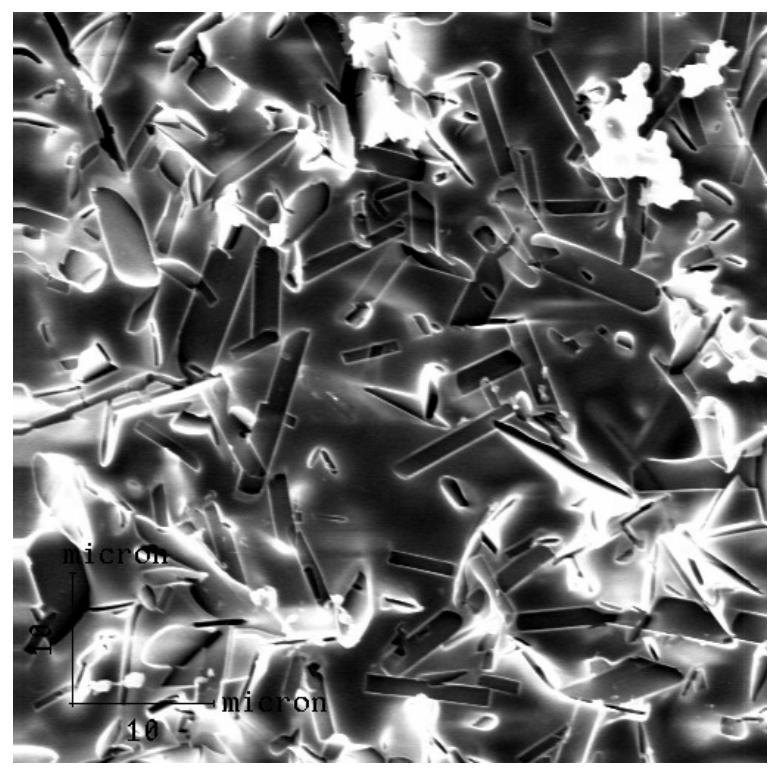

FIGURA 4 - MORFOLOGIA DO ÁCIDO BENZÓICO MICROPARTICULADO

A 120 BAR E $46,9^{\circ} \mathrm{C}$

As Tabelas 2 e 3 mostram o crescimento dos microrganismos durante os períodos de contagem, expressos em Unidades Formadoras de Colônia por mililitro de amostra (UFC/mL). Cada intervalo de contagem correspondeu a 7 dias que totalizaram o período de análise de 21 dias. 
$\mathrm{UFC} / \mathrm{mL}$

$\begin{array}{ccccc}\text { Amostra em Branco } & \mathbf{0} & \mathbf{1} & \mathbf{2} & \mathbf{3} \\ \text { Ácido Benzóico Comercial } & 460 & 1220 & 13200 & 92000 \\ \text { Ácido Benzóico Micronizado } & 590 & 530 & 1250 & 12000 \\ & 500 & 450 & 510 & 10000\end{array}$

TABELA 3 - CRESCIMENTO MICROBIANO COM 0,02\% m/v DE CONSERVADOR

$\mathrm{UFC} / \mathrm{mL}$

Amostra em Branco

Ácido Benzóico Comercial

Ácido Benzóico Micronizado

\begin{tabular}{cccc}
\multicolumn{5}{c}{ Intervalos de Contagem } \\
\hline $\mathbf{0}$ & $\mathbf{1}$ & $\mathbf{2}$ & $\mathbf{3}$ \\
35 & 146000 & 196000 & 244000 \\
10 & 1000 & 5100 & 49000 \\
10 & 300 & 1650 & 31000
\end{tabular}

O crescimento microbiológico foi mais significativo na amostra em que não foi adicionado o conservador. As amostras que apresentaram menor crescimento foram as adicionadas de conservador nas concentrações de 0,01 e $0,02 \% \mathrm{~m} / \mathrm{v}$ de ácido benzóico microparticulado. Essa afirmação pode ser melhor observada nas Figuras 5 e 6.

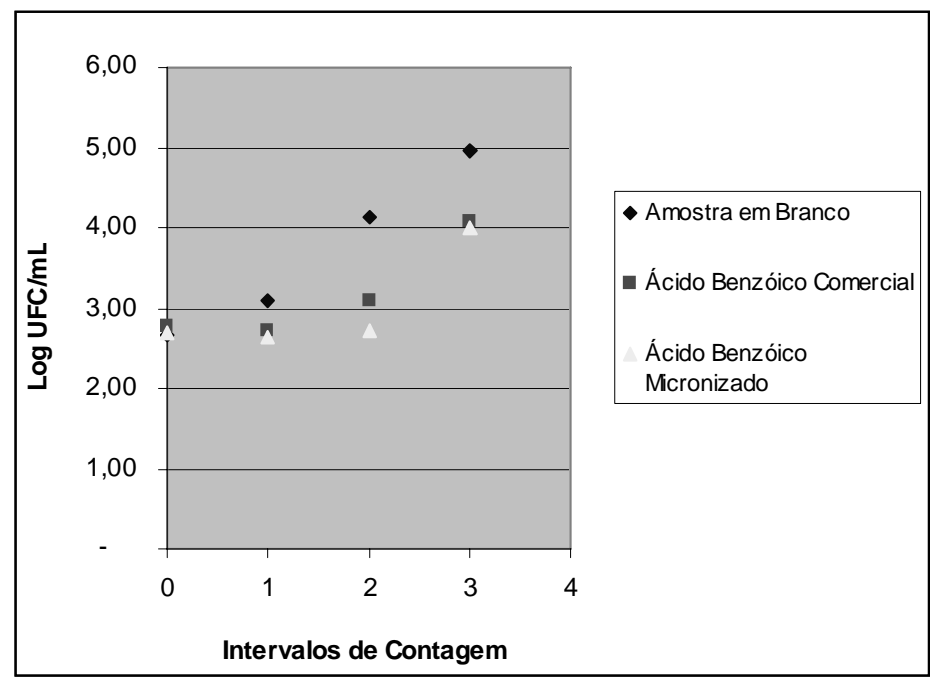

FIGURA 5 - CRESCIMENTO MICROBIOLÓGICO NA CONCENTRAÇÃO DE 0,01\% m/v

Os gráficos são expressos em unidades logarítmicas, pois além de ser a medida de contagem em análise microbiológica fornece melhor visualização do crescimento devido à proximidade das unidades.

Nos experimentos conduzidos com ácido benzóico na concentração de 0,01\% m/v pode-se observar que a amostra que continha o ácido benzóico microparticulado conservou durante os dois 
primeiros estágios de contagem o mesmo ciclo log de crescimento, causando assim retardo no início da fase exponencial do crescimento microbiano.

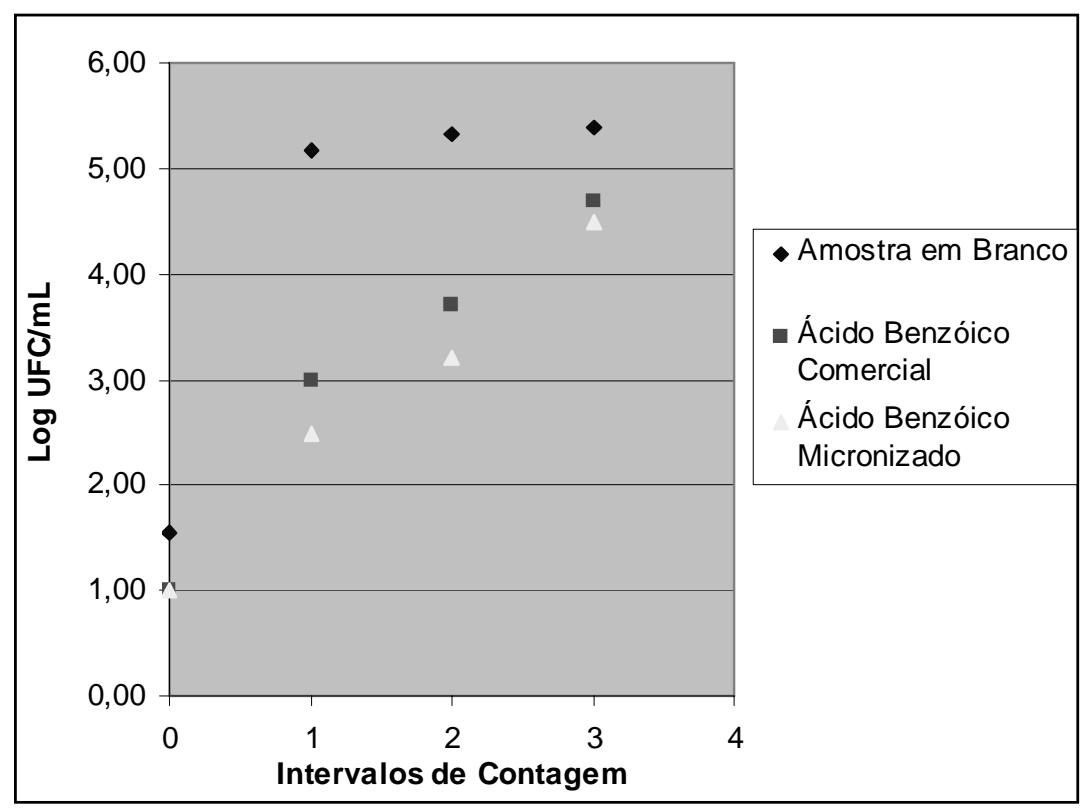

\section{FIGURA 6 - CRESCIMENTO MICROBIOLÓGICO NA CONCENTRAÇÃO DE 0,02\% M/V}

Os experimentos conduzidos com a concentração de $0,02 \% \mathrm{~m} / \mathrm{v}$ não retardaram o ciclo de crescimento, porém a fase de crescimento exponencial foi melhor controlada na amostra que continha o ácido benzóico microparticulado.

O melhor efeito do conservador microparticulado quando comparado ao comercial deve-se à sua maior solubilidade e, consequentemente, maior biodisponibilidade no meio em que está presente. Tal efeito foi observado por Alleman, Leroux e Gurny (1998) ao obterem micropartículas de compostos farmacêuticos pelo processo RESS.

No terceiro intervalo de contagem, o crescimento microbiano atingiu o mesmo ciclo logarítmico, para todas as amostras. Tal fato mostra que o conservador perdeu seu efeito e que seu tempo ótimo, independente da sua morfologia, foi de aproximadamente duas semanas.

Diversos fatores podem ter causado a ineficiência do conservador a partir do terceiro intervalo, como a adaptação dos microrganismos ao meio com conservador, ao seu consumo por completo pelos microrganismos devido à baixa concentração, ou por ter se combinado com outros compostos do suco.

\section{CONCLUSÃO}

Os resultados obtidos evidenciaram maior eficiência do ácido benzóico microparticulado como conservador de suco de laranja natural quando comparado com o produto comercial. A micronização de partículas pelo método RESS mostrou-se promissora para a produção de conservadores de alimentos.

Visando melhores resultados e informações mais precisas, os efeitos do ácido em outras concentrações ou sobre outros microrganismos deverão ser estudados. Nada se pode afirmar ainda sobre o seu efeito em concentrações mais elevadas, ou a respeito da concentração ótima para evitar o uso excessivo do conservador. 


\section{ABSTRACT \\ PRODUCTION IN SUPERCRITICAL CONDITIONS AND EVALUATION OF THE POTENTIAL OF THE MICRONIZED BENZOIC ACID IN FOODS}

The objective of this work was to evaluate the best production parameter of micronized benzoic acid by Rapid Expansion of Supercritical Solutions (RESS) and its effectiveness as an inhibitor of microorganisms on orange juice, comparing the obtained results to samples containing comercial benzoic acid, and samples without conservative by means of microbiological analysis. The results indicated that the temperature did not interfere significantly on the particles morphology and at 160 bar pressure the smaller particles were formed. Microbiological growth was higher at samples without conservative, and samples with 0.01 and $0.02 \% \mathrm{w} / \mathrm{v}$ of micronized benzoic acid had smaller growth that samples with same concentration of commercial benzoic acid. The results showed that the micronization of particles by RESS comes as a promising method to food conservatives production, although further studies must be realized to evaluate its effectiveness over other microorganisms and in different concentrations.

KEY-WORDS: CONSERVATIVES; RESS; MICRORGANISMS.

\section{REFERÊNCIAS}

1 ALlemAN, E.; LEROUX, J.; GURNY, R. Micronization of drugs. Advanced Drug Delivery Reviews, v. 34, p. 171189, 1998.

2 APHA. American Public Health Association. Compendium of methods for the microbiological examination of foods. $3^{\text {rd }}$ ed. Washington, D.C., 1992. p.1219.

3 ARAÚJO, J.M.A. Química de alimentos: teoria e prática. Viçosa: UFV Imprensa Universitária, 1995. 335 p.

4 BRASIL. Ministério da Saúde. Agência Nacional de Vigilância Sanitária. RDC n. 12, de 2 jan. 2001. Dispõe sobre os princípios gerais para o estabelecimento de critérios e padrões microbiológicos para alimentos. Disponível em: <http:/ /www.vigilanciasanitaria.gov.br/anvisa.html>. Acesso em: 22 fev. 2010.

5 CALVO, M. Aditivos alimentarios: propiedades y efectos sobre la salud. Zaragoza: Mira Editores, 1991.

6 DE LUCA, C.; PASSI, S.; QUATTRUCCI, E. Simultaneous determination of sorbic acid, benzoic acid and parabens in foods: a new gas chromatography-mass spectrometry technique adopted in a survey on Italian foods and beverages. Food Additives and Contaminants, v. 12, n. 1, p. 1-7, 1995

7 DOMINGO, C.; BERENDS, E.M.; ROSMALEN, G.M. Precipitation of ultrafine benzoic acid by expansion of a supercritical carbon dioxide solution through a porous plate nozzle. Journal of Crystal Growth, v. 166, p. 989995, 1999.

8 FENNEMA, O. R. Food Chemistry. $3^{\text {rd }}$ ed. Nova lorque: Marcel Decker,1997.

9 FRANÇA, B.B.; FREITAS, A.F.; MENDES, M.F.; COELHO, G.L.V. Obtenção de micropartículas de ácido benzóico através da expansão com dióxido de carbono supercrítico. In: CONGRESSO BRASILEIRO DE SISTEMAS PARTICULADOS, 31, 2004, Uberlândia-MG. Anais... Uberlândia: UFU, 2004.

10 GIESE, J. Antimicrobials: assuring food safety. Food Technology, v. 48, n. 6, p.101-110, 1994.

11 HELFGEN, B.; HILS, P.; HOLZKNECHT, C.H.; TÜRK, M.; SCHABER, K. Simulation of particle formation during the rapid expansion of supercritical solutions. Aerosol Science, v. 32, p. 295-319, 2001.

12 JAY, J.M. Modern food microbiology. $6^{\text {th }}$. ed. Gaithersburg, Maryland: Aspen Publishers, 2000.

13 LEITÃO, M.F.F. Conservadores em alimentos e fatores que afetam sua eficiência no controle de microrganismos. Coletânea do ITAL, Campinas, v. 20, n. 2, p.116-127, 1990.

14 LUCK, E. Conservación química de los alimentos. Zaragoza: Acribia, 1981, p. 142-145.

15 LUECK, E. Food applications of sorbic acid and its salts. Food Additives and Contaminants, v. 7, n. 1, p. 711-715, 1990.

16 TÜRK, M. Formation of small organic particles by RESS: experimental and theoretical investigations. Journal of Supercritical Fluids, v 15, p. 79-89, 1999. 
17 TÜRK, M.; HILS, P.; HELFGEN, B.; SCHABER, K.; MARTIN, H.J.; WAHL, M.A. Micronization of pharmaceutical substances by the Rapid Expansion of Supercritical Solutions (RESS): a promising method to improve bioavailability of poorly soluble pharmaceutical agents. Journal of Supercritical Fluids, v. 22, p. 75-84, 2001.

18 WALKER, R. Toxicity of ascorbic acid and sorbates. Food Additives and Contaminants, v. 7, n. 1, p. 671-676, 1990.

19 WHO: World Health Organization. Benzoic acid and sodium benzoate. Geneva, 2000. (Concise International Chemical Assessment, Document 26.) 